\title{
Interplay of Exciton Condensation and the Quantum Spin Hall Effect in InAs/GaSb Bilayers
}

\author{
D. I. Pikulin and T. Hyart \\ Instituut-Lorentz, Universiteit Leiden, P.O. Box 9506, 2300 RA Leiden, The Netherlands
}

(Received 13 November 2013; published 30 April 2014)

\begin{abstract}
We study the phase diagram of the inverted InAs/GaSb bilayer quantum wells. For a small tunneling amplitude between the layers, we find that the system is prone to the formation of an $s$-wave exciton condensate phase, where the spin structure of the order parameter is uniquely determined by the small spinorbit coupling arising from the bulk inversion asymmetry. The phase is topologically trivial and does not support edge transport. On the contrary, for a large tunneling amplitude, we obtain a topologically nontrivial quantum spin Hall insulator phase with a $p$-wave exciton order parameter, which enhances the hybridization gap. These topologically distinct insulators are separated by an insulating phase with spontaneously broken time-reversal symmetry. Close to the phase transition between the quantum spin Hall and time-reversal broken phases, the edge transport shows quantized conductance in small samples, whereas in long samples the mean free path associated with the backscattering at the edge is temperature independent, in agreement with recent experiments.
\end{abstract}

DOI: 10.1103/PhysRevLett.112.176403

PACS numbers: 71.10.Pm, 73.20.-r, 73.63.Hs

Introduction.-Two-dimensional quantum spin Hall (QSH) insulators are topologically distinguishable from conventional insulators due to a nontrivial topological invariant arising from band inversion [1-3]. The conducting and valence bands in QSH insulators are connected by gapless helical edge modes, which are protected against elastic backscattering from time-reversal symmetric perturbations. Recent experimental advances have revealed two materials, HgTe/CdTe [4-6] and InAs/GaSb [7,8] quantum wells, where the existence of helical edge states has been confirmed. In addition to the unique electrical properties arising due to edge modes [1,2,4-8], these materials in proximity to superconductors are interesting as a platform for Majorana zero modes [9,10] and fluxcontrolled quantum information processing $[11,12]$.

The recent observation of the QSH effect in InAs/GaSb bilayers [8] is theoretically puzzling, because conductance quantization was found up to a magnetic field on the order of $10 \mathrm{~T}$ in short samples. On the other hand, even in the absence of magnetic field the longitudinal resistance in long samples increased linearly with the device length. While inelastic processes can, in principle, give rise to a finite mean free path associated with the backscattering at the edge, the existing theoretical models [13-20] do not explain the observation that the mean free path was found to be temperature independent at least for a temperature range of $20 \mathrm{mK}$ to $4.2 \mathrm{~K}[8,21,22]$. Therefore, we expect that nonperturbative effects due to disorder or interactions beyond the existing approaches are important. In particular, the temperature-independent mean free path indicates that the dominating backscattering process might be an elastic one, which is allowed if time-reversal symmetry is either dynamically or spontaneously broken. While mechanisms resulting in dynamical time-reversal symmetry breaking have been proposed $[23,24]$, it is unlikely that they could account for the experimentally observed mean free path $\sim 4 \mu \mathrm{m}$.

In this Letter, we consider the influence of exciton condensation on the QSH effect in InAs/GaSb bilayers. While exciton condensation is theoretically predicted in inverted type II electron-hole bilayers [25-31], such as InAs/GaSb quantum wells, an unambiguous observation of a thermodynamically stable exciton condensate phase in these systems has been a long-standing problem. Indeed, so far the best-studied exciton condensate phase is the quantum Hall bilayer state at half-filled Landau levels [32,33], where the ability to separately contact the two layers has facilitated the possibility to probe the order parameter in terms of counterflow superfluidity along the layers and Josephsonlike tunneling between the layers [34-38]. Here, we go beyond the earlier theoretical models for exciton condensates [25,28-31] by studying the spin structure of the order parameter, when the relevant spin-orbit and tunneling terms for the InAs/ GaSb bilayers are taken into account. For small tunneling amplitude, we find a topologically trivial $s$-wave exciton condensate phase, whereas for relatively large tunneling we obtain a topologically nontrivial QSH insulator phase. These topologically distinct insulators are separated by an insulating phase with spontaneously broken timereversal symmetry. Close to the phase transition between the QSH and time-reversal broken phases, the conductance is quantized in small samples, whereas the mean free path in long samples is temperature independent for a wide range of temperature, in agreement with the recent experiments [8].

Model.-We consider bilayer electron-hole systems described by Hamiltonian $\hat{H}=\hat{H}_{0}+\hat{H}_{I}$, where $\hat{H}_{0}$ is the single-particle Hamiltonian for the InAs/GaSb 
quantum wells (see below) and the Coulomb interaction between the electrons is described by the Hamiltonian

$$
\hat{H}_{I}=\frac{1}{2} \sum_{a, a^{\prime}, s, s^{\prime}} \sum_{\mathbf{k}, \mathbf{k}^{\prime}, \mathbf{q}} V^{a a^{\prime}}(q) c_{\mathbf{k} s a}^{\dagger} c_{\mathbf{k}^{\prime} s^{\prime} a^{\prime}}^{\dagger} c_{\mathbf{k}^{\prime}+\mathbf{q} s^{\prime} a^{\prime}} c_{\mathbf{k}-\mathbf{q} s a},
$$

where $V^{a a}(q)=e^{2} F^{a a}(q) /\left(2 \epsilon \epsilon_{0} L^{2} q\right), V^{12}(q)=V^{21}(q)=$ $e^{2} F^{12}(q) e^{-q d} /\left(2 \epsilon \epsilon_{0} L^{2} q\right)$, and $F^{a b}$ are the structure factors for the layers of thicknesses $W_{1,2}$, see Ref. [29].

By performing a mean-field approximation for the interaction term $\hat{H}_{I}$ and including the terms of the single-particle Hamiltonian $\hat{H}_{0}$ [3], we arrive to a mean-field Hamiltonian

$$
\begin{aligned}
\hat{H}_{\mathrm{mf}} & =\sum_{\mathbf{k}} \psi_{\mathbf{k}}^{\dagger} H(\mathbf{k}) \psi_{\mathbf{k}}, \\
H(\mathbf{k}) & =\left(\begin{array}{ll}
H_{11}(\mathbf{k}) & H_{12}(\mathbf{k}) \\
H_{12}^{\dagger}(\mathbf{k}) & H_{22}(\mathbf{k})
\end{array}\right),
\end{aligned}
$$

where the Hamiltonian for each layer is given by

$$
\begin{aligned}
H_{11}(\mathbf{k})= & {\left[\frac{\hbar^{2} k^{2}}{2 m_{e}}-E_{G}+\epsilon_{1}^{\mathrm{mf}}(\mathbf{k})-\mu\right] \sigma_{0}-\sum_{i=1}^{3} h_{1, i}^{\mathrm{mf}}(\mathbf{k}) \sigma_{i} } \\
& +\Delta_{e}\left(k_{x} \sigma_{1}-k_{y} \sigma_{2}\right)+\xi_{e}\left(k_{y} \sigma_{1}-k_{x} \sigma_{2}\right) \\
H_{22}(\mathbf{k})= & {\left[E_{G}-\frac{\hbar^{2} k^{2}}{2 m_{h}}+\epsilon_{2}^{\mathrm{mf}}(\mathbf{k})-\mu\right] \sigma_{0}-\sum_{i=1}^{3} h_{2, i}^{\mathrm{mf}}(\mathbf{k}) \sigma_{i} } \\
& +\Delta_{h}\left(k_{x} \sigma_{1}+k_{y} \sigma_{2}\right)
\end{aligned}
$$

and the coupling between the layers is described by

$$
H_{12}(\mathbf{k})=A\left(k_{x} \sigma_{3}+i k_{y} \sigma_{0}\right)-i \Delta_{z} \sigma_{2}-\Delta^{\mathrm{mf}}(\mathbf{k}) .
$$

Here, $E_{G}$ is the inverted band gap, $\mu$ is the chemical potential, and $m_{e(h)}$ are the effective masses. Because of the $s$-and $p$-like natures of the conduction and valence bands, respectively, the tunneling term $A\left(k_{x} \sigma_{3}+i k_{y} \sigma_{0}\right)$ must be odd in momentum. The spin-orbit couplings $\Delta_{e}$, $\Delta_{h}$, and $\Delta_{z}$ arise due to bulk inversion asymmetry, and $\xi_{e}$ is the Rashba coupling. The mean-field potentials should be solved self-consistently from equations [31]

$$
\begin{gathered}
\epsilon_{a}^{\mathrm{mf}}(\mathbf{k})=-\frac{1}{2} \sum_{s, \mathbf{k}^{\prime}} V^{a a}\left(\mathbf{k}-\mathbf{k}^{\prime}\right)\left[\rho_{s s}^{a a}\left(\mathbf{k}^{\prime}\right)-\rho_{0}^{a a}\left(\mathbf{k}^{\prime}\right)\right], \\
\mathbf{h}_{a}^{\mathrm{mf}}(\mathbf{k})=\frac{1}{2} \sum_{s, s^{\prime}, \mathbf{k}^{\prime}} V^{a a}\left(\mathbf{k}-\mathbf{k}^{\prime}\right) \rho_{s s^{\prime}}^{a a}\left(\mathbf{k}^{\prime}\right) \vec{\sigma}_{s, s^{\prime}}
\end{gathered}
$$

and

$$
\Delta_{s, s^{\prime}}^{\mathrm{mf}}=\sum_{\mathbf{k}^{\prime}} V^{12}\left(\mathbf{k}-\mathbf{k}^{\prime}\right) \rho_{s^{\prime} s}^{21}\left(\mathbf{k}^{\prime}\right) .
$$

Here, $\rho_{s s^{\prime}}^{a a^{\prime}}(\mathbf{k})=\left\langle c_{\mathbf{k} s a}^{\dagger} c_{\mathbf{k} s^{\prime} a^{\prime}}\right\rangle$ is the Hartree-Fock density matrix and $\rho_{0}^{a a}(\mathbf{k})$ is the density matrix for full valence band in the hole layer and empty conduction band in the electron layer [31]. The mean-field potentials $\epsilon_{a}^{\mathrm{mf}}(\mathbf{k})$ describe the renormalization of the band structure, whereas $\mathbf{h}_{a}^{\mathrm{mf}}(\mathbf{k})$ can account for spontaneous magnetization and the renormalization of the spin-orbit couplings. For our purposes, the most interesting mean-field potentials are $\Delta^{\mathrm{mf}}(\mathbf{k})=\sum_{i=0}^{4} \Delta_{i}^{\mathrm{mf}}(\mathbf{k}) \sigma_{i}$, which describe the full spin structure of the exciton condensate order parameter.

The natural length $d_{0}$ and energy $E_{0}$ scales of the problem can be determined from the relation $E_{0}=$ $\left(m_{e}^{-1}+m_{h}^{-1}\right) \hbar^{2} / 2 d_{0}^{2}=e^{2} /\left(4 \pi \epsilon \epsilon_{0} d_{0}\right)$. For $\mathrm{InAs} / \mathrm{GaSb}$ bilayers, typical parameters in the regime of band inversion are expected to be $E_{0} / k_{B} \sim 100 \mathrm{~K}, d_{0} \sim 10 \mathrm{~nm}, m_{e} / m_{h} \sim 1$, $A /\left(E_{0} d_{0}\right) \sim 0.1, \Delta_{z} / E_{0} \sim 0.01, \xi_{e} /\left(E_{0} d_{0}\right) \sim-0.1, d / d_{0} \ll 1$, $W_{a} / d_{0} \sim 1$, and $\Delta_{e}, \Delta_{h} \sim 0.001$ [39]. The parameters $E_{G}$ and $\mu$ describe the densities in the layers and can be controlled with gate voltages. The tunneling terms $A$ and $\Delta_{z}$ are exponentially sensitive to the width and height of an insulating barrier between the layers.

Results.-For $A=\Delta_{z}=\Delta_{e}=\Delta_{h}=\xi_{e}=\mu=0$, the only nonzero mean-field potentials are $\epsilon^{\mathrm{mf}}(\mathbf{k})$ and $\Delta^{\mathrm{mf}}(\mathbf{k})$. The main effect of $\epsilon^{\mathrm{mf}}(\mathbf{k})$ is the renormalization of $E_{G}$ to $E_{G}^{R}$. Because the densities are controlled by the gate voltages, we express our results in terms of $E_{G}^{R}$. For realistic densities of electrons and holes, the system undergoes a second-order phase transition as a function of temperature, and below the critical temperature $T_{c} \sim$ $0.1 E_{0} / k_{B}$ an $s$-wave exciton condensate order parameter $\Delta^{\mathrm{mf}}(\mathbf{k})$ appears due to spontaneous symmetry breaking. Because the Hamiltonian has a lot of symmetries in the absence of tunneling and spin-orbit couplings, all timereversal symmetric $s$-wave order parameters $\Delta^{\mathrm{mf}}(\mathbf{k})$ with equal total magnitude $\sum_{i=0}^{4}\left|\Delta_{i}^{\mathrm{mf}}(\mathbf{k})\right|^{2}$ are degenerate solutions of the problem.

The two most important parameters concerning the exciton order parameter are the tunnel couplings $A$ and $\Delta_{z}$, because they act as symmetry-breaking terms in the Hamiltonian turning the second-order phase transition to the exciton condensate phase into a crossover. It is intuitively clear that $\Delta_{z}$ and $A$ favor an even-parity exciton condensate order parameter $i \Delta_{s} \sigma_{2}$ and an odd-parity exciton order parameter $-\Delta_{p}\left(k_{x} \sigma_{3}+i k_{y} \sigma_{0}\right) \quad\left(\Delta_{s}\right.$, $\left.\Delta_{p} \in \mathbb{R}\right)$, respectively. Therefore, there is a competition between even- and odd-parity exciton condensate that can be described by studying the parity of order parameter

$$
\mathcal{P}=\frac{\Delta_{\mathrm{tot}}^{\mathrm{even}}-\Delta_{\mathrm{tot}}^{\mathrm{odd}}}{\Delta_{\mathrm{tot}}^{\mathrm{even}}+\Delta_{\mathrm{tot}}^{\mathrm{odd}}}
$$

where $\quad \Delta_{\text {tot }}^{\text {even }}=\sqrt{\int d k k \sum_{i}\left|\Delta_{i, 0}^{\mathrm{mf}}(k)\right|^{2}} \quad$ and $\quad \Delta_{\mathrm{tot}}^{\mathrm{odd}}=$ $\sqrt{\int d k k \sum_{i, n= \pm 1}\left|\Delta_{i, n}^{\mathrm{mf}}(k)\right|^{2}}$ are obtained using expansion $\Delta_{i}^{\mathrm{mf}}(\mathbf{k})=\sum_{n} \Delta_{i, n}^{\mathrm{mf}}(k) e^{i n \theta_{k}}$ in terms of azimuthal angle $\theta_{k}$ in momentum space. We obtain the phase diagram in parameters $A$ and $E_{G}^{R}$ as they can be changed in a controlled way in the experiments. For small $A$, we find a topologically 

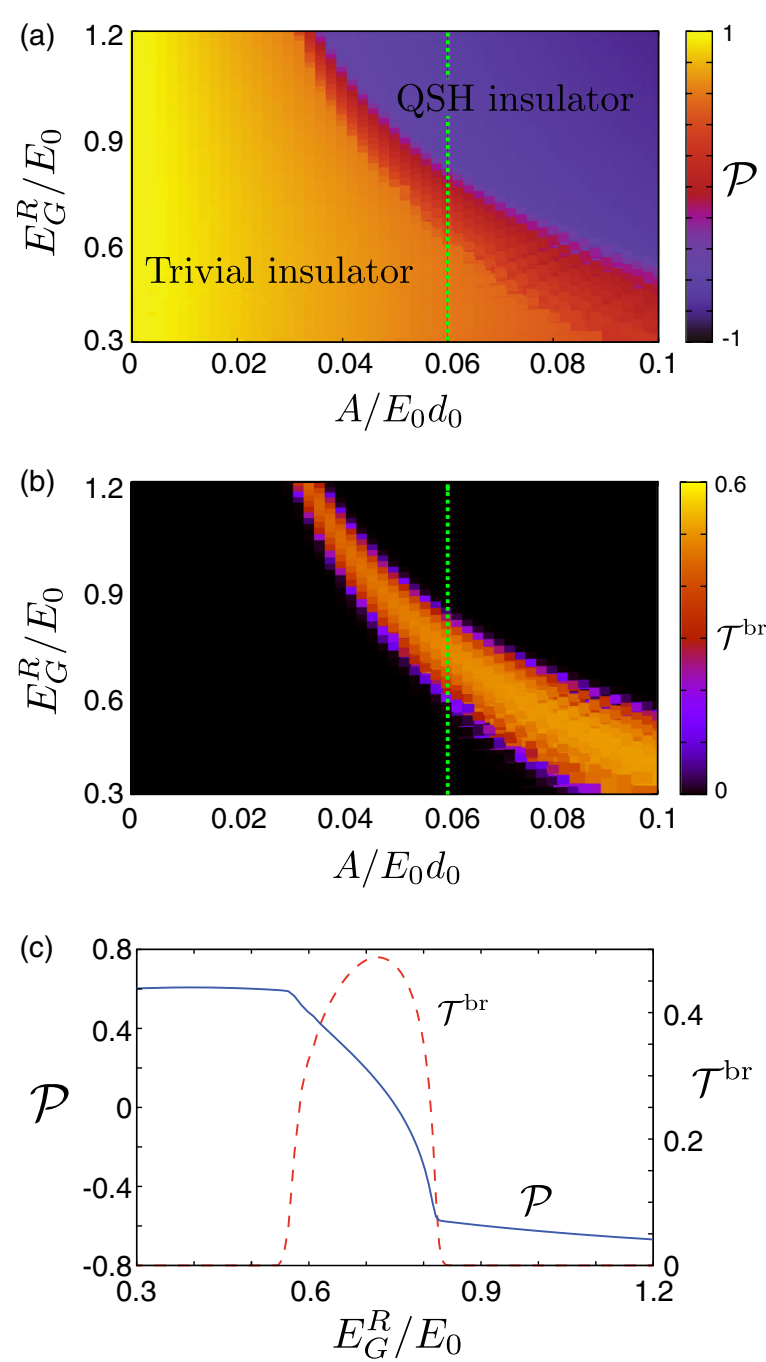

FIG. 1 (color online). (a) Parity of order parameter $\mathcal{P}$ as a function of $E_{G}^{R}$ and $A$ for $\Delta_{z} / E_{0}=0.02, m_{e} / m_{h}=1$, $W_{1,2} / d_{0}=0.8$, and $\mu=d=\Delta_{e}=\Delta_{h}=\xi_{e}=0$. (b) The time-reversal symmetry-breaking order parameter $\mathcal{T}^{\text {br }}$ as a function of $E_{G}^{R}$ and $A$ for the same parameters. (c) Line cut showing $\mathcal{P}$ and $\mathcal{T}^{\text {br }}$ as a function of $E_{G}^{R}$ for $A / E_{0} d_{0}=0.06$. The insulating phase with broken time-reversal symmetry is separated from the trivial and QSH insulators by second-order phase transitions.
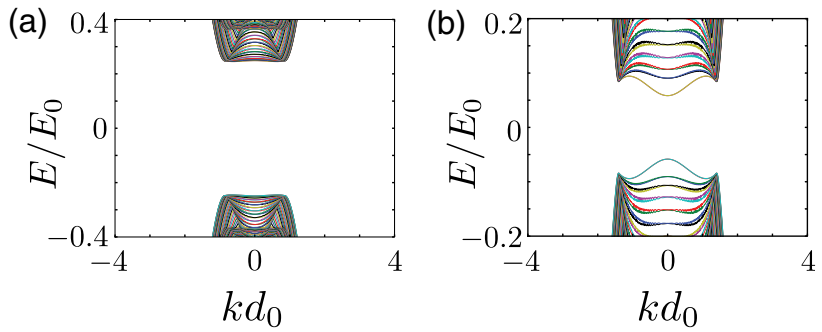

trivial $s$-wave exciton condensate phase, whereas for large $A$ we obtain a topologically nontrivial QSH insulator phase with a $p$-wave exciton order parameter [Fig. 1(a)], in agreement with our expectations. Interestingly, we find that these phases are separated by an insulating phase with spontaneously broken time-reversal symmetry. This timereversal symmetry broken phase is shown in Fig. 1(b), where we have characterized the time-reversal symmetry breaking with a parameter

$$
\mathcal{T}^{\mathrm{br}}=\frac{\Delta_{\mathrm{tot}}^{\mathrm{br}}}{\Delta_{\mathrm{tot}}^{\mathrm{ts}}+\Delta_{\mathrm{tot}}^{\mathrm{br}}}
$$

Here, the relative strength of the order parameter obeying the time-reversal symmetry is defined as

$$
\begin{aligned}
\left(\Delta_{\mathrm{tot}}^{\mathrm{ts}}\right)^{2}= & \int d k k\left\{\left(\Re \Delta_{0,0}^{\mathrm{mf}}\right)^{2}+\frac{\left(\Re \Delta_{0,1}^{\mathrm{mf}}-\Re \Delta_{0,-1}^{\mathrm{mf}}\right)^{2}}{2}\right. \\
& +\frac{\left(\Im \Delta_{0,1}^{\mathrm{mf}}+\Im \Delta_{0,-1}^{\mathrm{mf}}\right)^{2}}{2} \\
& +\sum_{i=1}^{3}\left[\left(\Im \Delta_{i, 0}^{\mathrm{mf}}\right)^{2}+\frac{\left(\Re \Delta_{i, 1}^{\mathrm{mf}}+\Re \Delta_{i,-1}^{\mathrm{mf}}\right)^{2}}{2}\right. \\
& \left.\left.+\frac{\left(\Im \Delta_{i, 1}^{\mathrm{mf}}-\Im \Delta_{i,-1}^{\mathrm{mf}}\right)^{2}}{2}\right]\right\},
\end{aligned}
$$

and the strength of time-reversal symmetry-breaking $\Delta_{\mathrm{tot}}^{\mathrm{br}}$ can be calculated by interchanging the real $\Re \Delta_{i, n}^{\mathrm{mf}}$ and imaginary parts $\Im \Delta_{i, n}^{\mathrm{mf}}$ of the order parameter in this equation (see the Supplemental Material [40]). Secondorder phase transitions are clearly seen at the two boundaries of the time-reversal symmetry broken phase [Fig. 1(c)]. We have numerically confirmed that our results are valid for chemical potentials $|\mu| / E_{0} \lesssim 0.05$. With increasing $|\mu|$, the difference between the densities of the electrons and holes increases, and the preference for Fermi surface nesting gives rise to magnetization $[31,41,42]$. Our results are robust against including small spin-orbit coupling $\xi_{e} /\left(E_{0} d_{0}\right)=-0.07$ and asymmetry of effective masses $m_{e} / m_{h}=0.84$ that are expected to be present in InAs $/ \mathrm{GaSb}$ bilayers [39], but the locations of the phase boundaries depend strongly on the parameter $\Delta_{z}$ (see the Supplemental Material [40]). This situation should be contrasted to
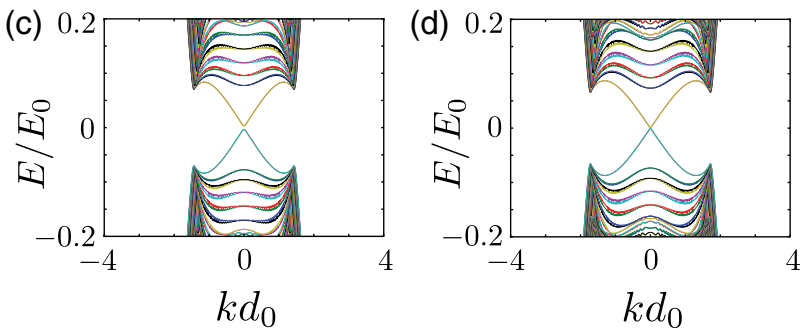

FIG. 2 (color online). Band structures for (a) $E_{G}^{R}=0.3 E_{0}$, (b) $E_{G}^{R}=0.78 E_{0}$, (c) $E_{G}^{R}=0.83 E_{0}$, and (d) $E_{G}^{R}=1.11 E_{0}$ and the other parameters same as in Fig. 1(c). Protected helical edge states appear only in the QSH phase. By decreasing $E_{G}^{R}$, the time-reversal symmetry breaking opens a gap in the edge state spectrum, and finally the edge states disappear when one approaches the trivial phase. 
$\mathrm{HgTe} / \mathrm{CdTe}$ QSH insulators described by the same Hamiltonian [2], where the exciton condensation does not give rise to phase transitions, because the conduction and valence bands are localized in the same quantum well so that $A$ is an order of magnitude larger than in InAs/GaSb bilayers [39].

Our numerical results can also be interpreted in the light of Ginzburg-Landau theory, which is obtained by expressing the exciton order parameter as $\Delta^{\mathrm{mf}}(\mathbf{k})=$ $i \Delta_{s} e^{i \phi_{s}} \sigma_{2}-\Delta_{p} e^{i \phi_{p}}\left(k_{x} \sigma_{3}+i k_{y} \sigma_{0}\right)$ and expanding the free-energy perturbatively using the tunnel couplings and exciton order parameter as a perturbation. We find that, similar to the case in Ref. [30], the lowest order terms in the free energy are proportional to $-\Delta_{z} \Delta_{s} \cos \phi_{s}$ and $-A \Delta_{p} \cos \phi_{p}$ favoring the order parameter with $\phi_{s}=$ $\phi_{p}=0$. On the other hand, the fourth-order expansion contains terms that are proportional to different combinations of $\Delta_{z}, A, \Delta_{s}$, and $\Delta_{p}$. These terms try to twist the phases of the order parameters $\phi_{s}$ and $\phi_{p}$ away from zero and are, thus, responsible for the spontaneous breaking of the time-reversal symmetry in the regime of the phase diagram where both $s$ - and $p$-wave order parameters are simultaneously large.

Transport.-To calculate the influence of the exciton condensation on the transport properties of the QSH insulator, we perform a $k \cdot p$ expansion of the mean-field potentials and calculate the conductance using a tightbinding Hamiltonian constructed from the resulting continuum model. The band structures in different parameter regimes are shown in Fig. 2, and the results for the disorderaveraged differential conductance $\langle G\rangle$ are shown in Fig. 3. In the QSH regime, the edge states are protected from elastic backscattering, and therefore, we find perfect conductance quantization for all disorder strengths $V_{\text {dis }}$ shown in the figure. On the other hand, in the regime of weakly broken time-reversal symmetry, the Born approximation gives a mean free path $\ell=a 4 \hbar^{4} v^{4} k_{F}^{2} /\left(V_{\mathrm{dis}}^{2} \xi \Delta_{\mathrm{br}}^{2}\right)$, where we have assumed uncorrelated disorder potential $\left\langle V(x) V\left(x^{\prime}\right)\right\rangle=V_{\text {dis }}^{2} \xi \delta\left(x-x^{\prime}\right)$ along the edge, $\Delta_{\text {br }}$ is the energy gap in the edge state spectrum due to the timereversal symmetry-breaking order parameter, and $a \sim 1$ is a fitting parameter, which depends on the detailed structure of the edge states [43]. Our numerical results for $\langle G\rangle$ are in good agreement with the Born approximation (Fig. 3). Importantly, the mechanism of elastic scattering due to the spontaneous breaking of the time-reversal symmetry remains effective for $T \ll T_{c}$. Since typically $T_{c} \sim 10 \mathrm{~K}$, and we estimate that $\ell \sim 4 \mu \mathrm{m}$ already for a reasonably weak disorder, we conclude that this mechanism is a viable candidate for the explanation of the temperature-independent mean free path observed in recent experiments $[8,21,22]$. We also predict that the resistance is peaked at the crossing point of the edge state spectrum-in agreement with the recent experiment [21], where the maximum resistance was observed deep inside the topological gap.

Summary and discussion.-In summary, we have studied the exciton condensation in inverted electron-hole bilayers,

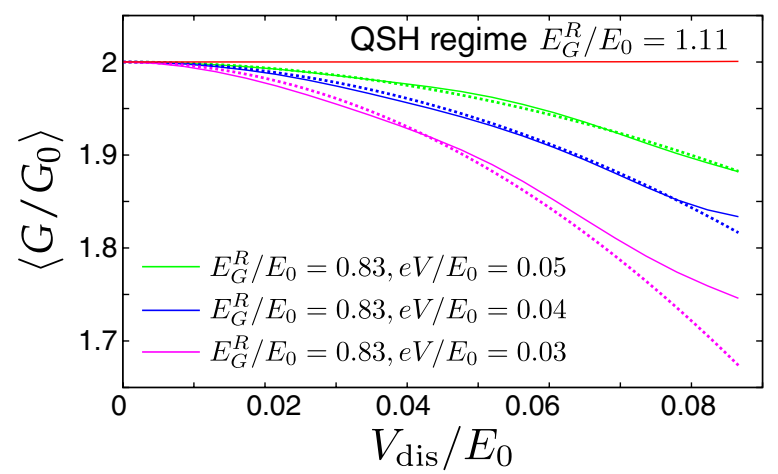

FIG. 3 (color online). Disorder-averaged differential conductance $\langle G\rangle$ as a function of $V_{\text {dis }}$ for a device with length $L=100 d_{0}$ and different values of $E_{G}^{R}$ and voltage $e V$. In the QSH regime $E_{G}^{R}=1.11 E_{0}$, the conductance is quantized to $G=2 G_{0}$ $\left(G_{0}=e^{2} / h\right)$. In the regime of weakly broken time-reversal symmetry $E_{G}^{R}=0.83 E_{0}$, numerically calculated $\langle G\rangle$ (thick lines) are in agreement with $G=2 G_{0}(1-L / \ell)$ (dashed lines), where $\ell$ is obtained from Born approximation with a fitting parameter $a=3.1$. The other parameters are the same as in Fig. 1(c).

where the $s$-like conduction band and $p$-like hole band are localized in different quantum well layers. We have calculated the phase diagrams, which show competition between a topologically trivial $s$-wave exciton condensate phase and a nontrivial QSH phase. These topologically distinct phases are separated by an insulating phase with spontaneously broken time-reversal symmetry, which is energetically favored, because it keeps the system gapped when it experiences a transition between the topologically distinct insulators. Our results can explain the unexpected temperature-independent mean free path observed in InAs/GaSb bilayers [8].

We also point out that a more detailed experimental study can confirm that the backscattering at the edge happens due to the spontaneous time-reversal symmetry breaking. The phase diagram we discuss can be studied as a function of the tunneling amplitudes by controlling the width and height of an insulating barrier between the layers [33], and the exciton order parameter can be probed via the collective modes and vortex excitations. In quantum Hall bilayers, the exciton order parameter has been studied in terms of counterflow superfluidity and Josephson-like tunneling anomaly [34-38], and there these properties are known to be strongly influenced by disorder-induced fractionally charged vortices [44-55]. A controllable way to open an energy gap in the edge state spectrum by tuning the gate voltage may also be useful for studying Majorana zero modes and for electronic applications.

The conductance and band structures were calculated using KWANT [56]. We have benefited from discussions with I. Knez and C. W. J. Beenakker. This work was supported by the Foundation for Fundamental Research on Matter (FOM), the Netherlands Organization for Scientific Research (NWO/ OCW), and the European Research Council. 
Note added.-After the submission of this work, an independent work by Budich et al. appeared [57], where helical exciton condensates were considered in $\mathrm{HgTe}$ bilayers.

[1] C. L. Kane and E. J. Mele, Phys. Rev. Lett. 95, 226801 (2005).

[2] B. A. Bernevig, T. L. Hughes, and S.-C. Zhang, Science 314, 1757 (2006).

[3] C. Liu, T. L. Hughes, X.-L. Qi, K. Wang, and S.-C. Zhang, Phys. Rev. Lett. 100, 236601 (2008).

[4] M. König, S. Wiedmann, C. Brüne, A. Roth, H. Buhmann, L. W. Molenkamp, X.-L. Qi, and S.-C. Zhang, Science 318, 766 (2007).

[5] A. Roth, C. Brüne, H. Buhmann, L. W. Molenkamp, J. Maciejko, X.-L. Qi, and S.-C. Zhang, Science 325, 294 (2009).

[6] M. König, M. Baenninger, A. G. F. Garcia, N. Harjee, B. L. Pruitt, C. Ames, P. Leubner, C. Brüne, H. Buhmann, L. W. Molenkamp, and D. Goldhaber-Gordon, Phys. Rev. X 3, 021003 (2013).

[7] I. Knez, R.-R. Du, and G. Sullivan, Phys. Rev. Lett. 107, 136603 (2011).

[8] L. Du, I. Knez, G. Sullivan, and R.-R. Du, arXiv:1306.1925.

[9] L. Fu and C. L. Kane, Phys. Rev. Lett. 100, 096407 (2008).

[10] L. Fu and C. L. Kane, Phys. Rev. B 79, 161408(R) (2009).

[11] S. Mi, D. I. Pikulin, M. Wimmer, and C. W. J. Beenakker, Phys. Rev. B 87, 241405(R) (2013).

[12] T. Hyart, B. van Heck, I. C. Fulga, M. Burrello, A. R. Akhmerov, and C. W. J. Beenakker, Phys. Rev. B 88, 035121 (2013).

[13] J. Maciejko, C. Liu, Y. Oreg, X.-L. Qi, C. Wu, and S.-C. Zhang, Phys. Rev. Lett. 102, 256803 (2009).

[14] Y. Tanaka, A. Furusaki, and K. A. Matveev, Phys. Rev. Lett. 106, 236402 (2011).

[15] J. C. Budich, F. Dolcini, P. Recher, and B. Trauzettel, Phys. Rev. Lett. 108, 086602 (2012).

[16] F. Crepin, J. C. Budich, F. Dolcini, P. Recher, and B. Trauzettel, Phys. Rev. B 86, 121106 (2012).

[17] N. Lezmy, Y. Oreg, and M. Berkooz, Phys. Rev. B 85, 235304 (2012).

[18] R. Ilan, J. Cayssol, J. H. Bardarson, and J. E. Moore, Phys. Rev. Lett. 109, 216602 (2012).

[19] T. L. Schmidt, S. Rachel, F. von Oppen, and L. I. Glazman, Phys. Rev. Lett. 108, 156402 (2012).

[20] J. I. Väyrynen, M. Goldstein, and L. I. Glazman, Phys. Rev. Lett. 110, 216402 (2013).

[21] I. Knez, C. T. Rettner, S.-H. Yang, S. S. P. Parkin, L. Du, R.-R. Du, and G. Sullivan, Phys. Rev. Lett. 112, 026602 (2014).

[22] E. M. Spanton, K. C. Nowack, L. Du, R.-R. Du, and K. A. Moler, arXiv:1401.1531.

[23] A. M. Lunde and G. Platero, Phys. Rev. B 86, 035112 (2012).

[24] A. Del Maestro, T. Hyart, and B. Rosenow, Phys. Rev. B 87, 165440 (2013).

[25] L. V. Keldysh and Yu. V. Kopaev, Fiz. Tverd. Tela 6, 2791 (1964) [Sov. Phys. Solid State 6, 2219 (1965)].

[26] Yu. E. Lozovik and V. I. Yudson, JETP Lett. 22, 274 (1975).

[27] Yu. E. Lozovik and V. I. Yudson, Sov. Phys. JETP 44, 389 (1976).
[28] X. Zhu, P. B. Littlewood, M. S. Hybertsen, and T. M. Rice, Phys. Rev. Lett. 74, 1633 (1995).

[29] Y. Naveh and B. Laikhtman, Phys. Rev. Lett. 77, 900 (1996).

[30] P. B. Littlewood and X. Zhu, Phys. Scr. T68, 56 (1996).

[31] Y.-P. Shim and A. H. MacDonald, Phys. Rev. B 79, 235329 (2009).

[32] H. A. Fertig, Phys. Rev. B 40, 1087 (1989).

[33] S. Q. Murphy, J. P. Eisenstein, G. S. Boebinger, L. N. Pfeiffer, and K. W. West, Phys. Rev. Lett. 72, 728 (1994).

[34] I. B. Spielman, J. P. Eisenstein, L. N. Pfeiffer, and K. W. West, Phys. Rev. Lett. 84, 5808 (2000).

[35] I. B. Spielman, J. P. Eisenstein, L. N. Pfeiffer, and K. W. West, Phys. Rev. Lett. 87, 036803 (2001).

[36] Y. Yoon, L. Tiemann, S. Schmult, W. Dietsche, K. von Klitzing, and W. Wegscheider, Phys. Rev. Lett. 104, 116802 (2010).

[37] A. D. K. Finck, J. P. Eisenstein, L. N. Pfeiffer, and K. W. West, Phys. Rev. Lett. 106, 236807 (2011).

[38] X. Huang, W. Dietsche, M. Hauser, and K. von Klitzing, Phys. Rev. Lett. 109, 156802 (2012).

[39] C. Liu and S.-C. Zhang, Models and materials for topological insulators (unpublished).

[40] See the Supplemental Material at http://link.aps.org/ supplemental/10.1103/PhysRevLett.112.176403 for a more detailed discussion on how the parameters $\mathcal{P}$ and $\mathcal{T}$ describe the topological index and the time-reversal symmetry breaking, respectively.

[41] B. A. Volkov, Yu. V. Kopaev, and A. I. Rusinov, Sov. Phys. JETP 41, 952 (1975).

[42] B. A. Volkov, A. I. Rusinov, and R. Kh. Timerov, Sov. Phys. JETP 43, 589 (1976).

[43] B. Zhou, H.-Z. Lu, R.-L. Chu, S.-Q. Shen, and Q. Niu, Phys. Rev. Lett. 101, 246807 (2008).

[44] K. Moon, H. Mori, K. Yang, S. M. Girvin, A. H. MacDonald, L. Zheng, D. Yoshioka, and S.-C. Zhang, Phys. Rev. B 51, 5138 (1995).

[45] K. Yang, K. Moon, L. Belkhir, H. Mori, S. M. Girvin, A. H. MacDonald, L. Zheng, and D. Yoshioka, Phys. Rev. B 54, 11644 (1996).

[46] A. Stern, S. M. Girvin, A. H. MacDonald, and N. Ma, Phys. Rev. Lett. 86, 1829 (2001).

[47] L. Balents and L. Radzihovsky, Phys. Rev. Lett. 86, 1825 (2001).

[48] M. M. Fogler and F. Wilczek, Phys. Rev. Lett. 86, 1833 (2001).

[49] T. Hyart and B. Rosenow, Phys. Rev. B 83, 155315 (2011).

[50] T. Hyart and B. Rosenow, Phys. Rev. Lett. 110, 076806 (2013).

[51] H. A. Fertig and J. P. Straley, Phys. Rev. Lett. 91, 046806 (2003).

[52] H. A. Fertig and G. Murthy, Phys. Rev. Lett. 95, 156802 (2005).

[53] P. R. Eastham, N. R. Cooper, and D. K. K. Lee, Phys. Rev. B 80, 045302 (2009).

[54] P. R. Eastham, N. R. Cooper, and D. K. K. Lee, Phys. Rev. Lett. 105, 236805 (2010).

[55] B. Seradjeh, J. E. Moore, and M. Franz, Phys. Rev. Lett. 103, 066402 (2009).

[56] C. W. Groth, M. Wimmer, A. R. Akhmerov, and X. Waintal, arXiv:1309.2926.

[57] J. Budich, B. Trauzettel, and P. Michetti, Phys. Rev. Lett. 112, 146405 (2014). 